\title{
Early skin immunological disturbance after Plasmodium-infected mosquito bites
}

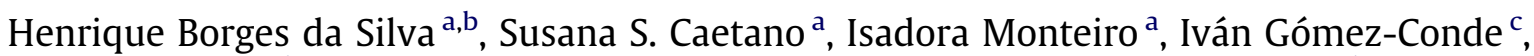 \\ Maria R. D'Imperio Lima ${ }^{\mathrm{b}}$, Carlos E. Tadokoro ${ }^{\mathrm{a}, *}$ \\ a Instituto Gulbenkian de Ciência, Oeiras 2780-156, Portugal \\ ${ }^{\mathrm{b}}$ Departamento de Imunologia, Instituto de Ciências Biomédicas, Universidade de São Paulo, São Paulo 05508-900, Brazil \\ ${ }^{\mathrm{c}}$ Escuela Superior de Ingeniería Informática, Universidade de Vigo, Ourense 32004, Spain \\ d Unidade de Malária, Instituto de Medicina Molecular, Universidade de Lisboa, Lisboa 1649-028, Portugal \\ e Departamento de Parasitologia, Instituto de Ciências Biomédicas, Universidade de São Paulo, São Paulo 05508-900, Brazil
} Kirsten Hanson $^{\mathrm{d}}$, Carlos Penha-Gonçalves ${ }^{\mathrm{a}}$, David N. Olivieri ${ }^{\mathrm{c}}$, Maria M. Mota ${ }^{\mathrm{d}}$, Cláudio R. Marinho ${ }^{\mathrm{e}}$,

\section{A R T I C L E I N F O}

\section{Article history:}

Received 9 April 2012

Accepted 8 June 2012

Available online $\mathrm{xxxx}$

\section{Keywords:}

Regulatory T cell

Plasmodium berghei

Dendritic cell

Skin

Immunity

\begin{abstract}
A B S T R A C T
Although the role of regulatory T cells (Tregs) during malaria infection has been studied extensively, such studies have focused exclusively on the role of Treg during the blood stage of infection; little is known about the detailed mechanisms of Tregs and sporozoite deposition in the dermis by mosquito bites. In this paper we show that sporozoites introduced into the skin by mosquito bites increase the mobility of skin Tregs and dendritic cells (DCs). We also show differences in MHC class II and/or CD86 expression on skin-resident dendritic cell subtypes and macrophages. From the observed decrease of the number of APCs into draining lymph nodes, suppression of CD28 expression in conventional CD4 T cells, and a low homeostatic proliferation of skin-migrated CD4 T found in nude mice indicate that Tregs may play a fundamental role during the initial phase of malaria parasite inoculation into the mammalian host.
\end{abstract}

(c) 2012 Elsevier Inc. All rights reserved.

\section{Introduction}

Understanding the detailed mechanisms of immune regulation during malaria infection is motivated by the large amount of affected people living in areas of high contamination risk. Indeed, approximately half the world's population lives in malaria-endemic areas, with an estimated 240 million clinical cases and nearly one million deaths annually [1]. While the mechanisms that lead to severe illness are not yet fully understood, the failure of the immune system to control the rapid parasitic replication together with the immunopathologic effects that are produced due to excessive inflammation, are important factors [2].

Regulatory T cells (Tregs) are key mediators for providing tolerance against self and non-self antigens $[3,4]$ as well as for curbing excessive immunological responses [5]. Whether in human and mouse hosts, or with different parasite strains, the nature and outcome of malaria infections are governed by the balance between Tregs and effector $\mathrm{T}$ cells (Teffs). Indeed, a high number of Tregs

Abbreviations: iRBCs, infected-Red Blood Cells; CM, Cerebral Malaria; YFP, Yellow Fluorescent Protein; INF, Infected; NI, Non-Infected.

* Corresponding author. Address: Instituto Gulbenkian de Ciência, Lab of Immune Regulation, R. da Quinta Grande 6, Oeiras 2780-156, Portugal. Fax: +351 21440 7970.

E-mail address: ctadokoro@igc.gulbenkian.pt (C.E. Tadokoro). during the acute phase may contribute to hyper-parasitemia, whereas a low number of Tregs could contribute to immunopathology during the chronic phase [6]. However, these experimental malaria studies were performed by infecting animals with iRBCs injected inside the peritoneal cavity, completely bypassing the pre-erythrocytic stages of the parasite life cycle. Thus, the immunological events that occur at the time of sporozoite injection or during the pre-erythrocytic phase of the infection, potentially dominated by Tregs, remain to be elucidated.

It is known that during the skin probing phase, when seeking blood vessels to feed, infected mosquitoes inject parasites into the dermis [7]. Part of these sporozoites enter into blood capillaries, while other parts stay within the dermis or migrate into the draining lymph nodes throughout the lymphatic vessels [8]. Presumably, transverse dermal cell movements by sporozoites (from the invasion of host cells that cause disruption of the plasma membrane) [9] are important for future establishment of liver stage infection, since it is the mechanism by which these parasites can escape from the dermal phagocytes [10]. More recently, it was reported that a small proportion of the dermal sporozoites can continue their development into merozoite forms (as they usually do inside liver cells) by infecting hair follicle cells [11]. Nonetheless, the contribution of these dermal merozoite forms compared to the entire host infection appears to be either inexistent or minimal [11]. Sporozoites injected by infected mosquitoes into actively 
immunized mice (animals pre-treated with irradiated sporozoites), or passively immunized animals (mice that received mAbs against sporozoites), stop moving and are rapidly destroyed [7,12], thereby inferring that immune system pre-activation by the first parasite inoculation can prevent future infections by new sporozoites.

Inside normal human skin, there are approximately twice as many $\mathrm{T}$ cells as found in blood. Moreover, $15-20 \%$ of these T cells are Tregs, since they express Foxp3 which is a master transcriptional regulator of Treg development and function [13,14]. Therefore, since the skin is constantly exposed to external injuries, the presence of high amount of Tregs in this body region may attenuate an inadequate immune response. In support of this hypothesis, Scurfy-like mice (Foxp3-deficient animals) develop inflammatory lesions within the skin [15].

In this study, we evaluated initial DC and Treg behavior that occur within the dermis after mosquito bites. Our results indicate that the parasite injection rapidly triggers motility of skin DCs and Tregs as well as suppression of MHC class II and CD86 on antigen presenting cells. Just after skin sporozoite infection ( $2 \mathrm{~h}$ later), we observed a strong decrease in the amount of APC inside the draining lymph nodes, as well as a suppression of CD28 expression on conventional CD4 T cells, thereby indicating a compromise of their activation state. Finally, from CD4 T cell counts in lymphoid organs 14 days after skin transplantation, we could indirectly infer the Treg suppression effect of homeostatic proliferation. Our results clearly show that non-Treg cells from infected mosquito bitten skins proliferate less inside the recipient host than recipients of non-infected mosquito bitten skins. Thus, we suggest that these early mechanisms associated with Tregs and DCs strongly influence the establishment of the parasite during the infection.

\section{Material and methods}

\subsection{Animals and parasites}

In this study we used Foxp3-KI ${ }^{\text {gfp/gfp }}$ (Foxp3-GFP), CD11c-YFP transgenic (CD11C-YFP), and C57Bl/10.PL (B10.PL) animals. All animals were produced at the IGC animal facility, backcrossed with the B10.PL background, and used 6 weeks from birth. Anopheles stephensi mosquitoes infected with Plasmodium berghei ANKA were produced by "Unidade de Malária, Instituto de Medicina Molecular, Universidade de Lisboa", Lisbon, Portugal. All procedures were approved by the Institutional Animal Care and Use Committee (IACUC) of the Instituto Gulbenkian de Ciência (IGC) and were in agreement with the Federation of European Laboratory Animal Science Associations (FELASA) directives. The approval ID number of this project is $A 010 / 2010$.

\subsection{Intravital imaging of dermal GFP-Tregs and/or YFP-dendritic cells (YFP-DCS)}

Foxp3-GFP and/or CD11c-YFP mice were deeply anesthetized with $1.2 \mathrm{mg}$ ketamine (Imalgene 1000, Merial) and $0.019 \mu \mathrm{g}$ xylazine (Rompun $2 \%$, Bayer) per $22 \mathrm{~g}$ of mouse weight. Subsequently, we allowed non-infected or $P$. berghei ANKA-infected mosquitoes to feed on the ears of these animals for 1 or $5 \mathrm{~min}$. The average parasite load per mosquito salivary gland was approximately 30,000 sporozoites. We estimate that one to two mosquitoes had access to the ear to feed. After this feeding time, the animals were immediately immobilized on top of a heating pad at $37^{\circ} \mathrm{C}$, and the ear was fixed in a second heating pad to maintain the ear temperature between 25 and $27^{\circ} \mathrm{C}$, which is the same temperature of ears of non-manipulated animals measured inside the cage. Our study also consisted of control animals that were not bitten by mosquitoes. Intravital imaging acquisitions were performed at three distinct time points: $1 \mathrm{~min}, 5 \mathrm{~min}$, or $2 \mathrm{~h}$ after mosquito bites. For observation of cells, we used a Zeiss Stereo Lumar stereoscope (Zeiss Inc, Germany), with $150 \times$ magnifications in an Apo Lumar S1.2x FWD $47 \mathrm{~mm}$ objective. In this configuration, one image pixel corresponds to $0.866 \mu \mathrm{m}$. All images were acquired for periods of 20-30 min by Micro-manager v.1.3 software. All images were processed by ImageJ (NIH, USA). Extracted cell speed differences were evaluated by ANOVA statistics.

\subsection{Cell tracking}

For obtaining detailed cell motility and velocity information, we performed both manual and automatic cell tracking from images at each time point. For manual tracking, we used the ImageJ software (NIH, USA) by identifying and selecting bright spots in the images according to their relative size, brightness, and motility. From this, we manually extracted the entire discernible path of these spots in order to obtain the detailed trajectories and quantify both the track length and cell speed. Due to the high skin auto-fluorescence background intensity with low signal to noise ratio, we also developed an automated algorithm for detecting and tracking cell motility in order to remove doubts about missed or misclassified Treg tracks. Detailed results from both the manual and automated tracking are presented in the Supplementary Section. In the supporting information section, we provide technical details of the algorithm for the interested reader.

\subsection{Isolation and analysis of activation markers in leukocytes}

To acquire a larger number of skin leukocytes, abdominal and/ or back skins of animals were used instead of ear skins. The hairs of these regions were shaved with razor blades just before mosquito feedings. Four to five mosquitoes bit each skin region for $30 \mathrm{~min}$. Just after the mosquito bites, or $1.5 \mathrm{~h}$ later, skins were cut in $3 \times 4 \mathrm{~cm}$-wide strips and incubated dermal-side down on top of $0.5 \%$ trypsin for $45 \mathrm{~min}$ at $37^{\circ} \mathrm{C}$. The dermis sections were then separated from the epidermis using histological forceps and incubated in CRPMI and $0.25 \mathrm{mg} / \mathrm{ml}$ of DNase I (Sigma, USA) and $10 \mathrm{mg} / \mathrm{ml}$ of Collagenase D (Roche Inc, USA), for $1.5 \mathrm{~h}$ at $37^{\circ} \mathrm{C}$. These tissues were kept under vigorous agitation for $40 \mathrm{~min}$ at $37^{\circ} \mathrm{C}$. Finally, all dermis tissue samples were filtered in cell strainers (BD Falcon Inc.) and washed by centrifugation. In the experiments performed $1.5 \mathrm{~h}$ after mosquito bites, the draining lymph nodes were also harvested, homogenized, and washed by centrifugation.

Leukocytes were labeled with anti-CD4-PerCP, anti-CD8Alexa647, anti-CD11b-Cy5, anti-CD11b-biotin, anti-CD11c-PE, anti-CD25-Cy5, anti-CD28-biotin, anti-CD44-biotin, anti-CD45PE, anti-CD62L-biotin, anti-CD69-biotin, anti-CD80-biotin, antiCD86-biotin, anti-CTLA-4-biotin, anti-RT1B-PerCP (anti-MHC class II). A secondary labeling with SAv-FITC was employed, while SAv-APC was used for cases with biotin mAbs usage. To avoid contamination of CD8 T cells in our DC subtype analysis, we excluded all possible CD3+ cells on those gates. To evaluate cell death, 7amino-actinomycin D (7-AAD) was used together with staining. All mAbs, SAv, and 7-AAD were purchased by BD Biosciences, USA. Also, all samples were evaluated by flow cytometry in a FACScalibur or FACSaria machine (BD Biosciences, USA). Data analysis was performed using Flow Jo (Tree Star Inc., USA) and GraphPad Prism 5.01 (GraphPad, USA) software. Each of our quoted results is representative of at least three repetitions.

\subsection{Homeostatic proliferation to in vivo tests Treg function}

Homeostatic proliferation is controlled by Tregs [16]. In order to indirectly measure the suppressive capacity of skin-migrated 
Tregs, we performed a homeostatic proliferation assay, measuring the amount of CD4 T cells that could be found in spleen, mesenteric, and inguinal LNs of immune-compromised animals, 14 days after skin transplantations.

Skins measuring $2 \mathrm{~cm}^{2}$ and containing approximately 200-300 $\mathrm{T}$ cells were transplanted to nude animals from the control, the NI bite, or the INF bite groups. After 14 days, spleen and lymph nodes were removed, homogenized, and the total number of cells counted in a Neubauer chamber. The number of CD4 T cells was determined according to the percentage of these cells found by flow cytometry. Since nude animals lack CD4 T cells, the amount of cells found corresponds to the amount of cells that escape from the suppressive control by Tregs. We also labeled these suspensions with anti-CD69-biotin and anti-CD62L-biotin MAbs in order to evaluate their activation state. All these results were compared with freshly isolated cells from organs of Foxp3-GFP animals. Each result that we quote is representative of at least three independent experiments.

\subsection{Statistical analysis}

Two-way ANOVA and Student's $t$ tests were performed in order to test the statistical difference between the groups of our experimental study. These tests yield $p$ values $<0.05$, which confirm statistically significance of our results. In order to confirm the adequacy of using these hypothesis tests, we applied the D'Agostino and Pearson omnibus normality test to each group of our experimental data. This test confirmed, using accepted thresholds, that it is appropriate to consider our data drawn from normal distribution and therefore adequate to apply the statistical hypothesis tests as mentioned above.

A Time after mosquito bite

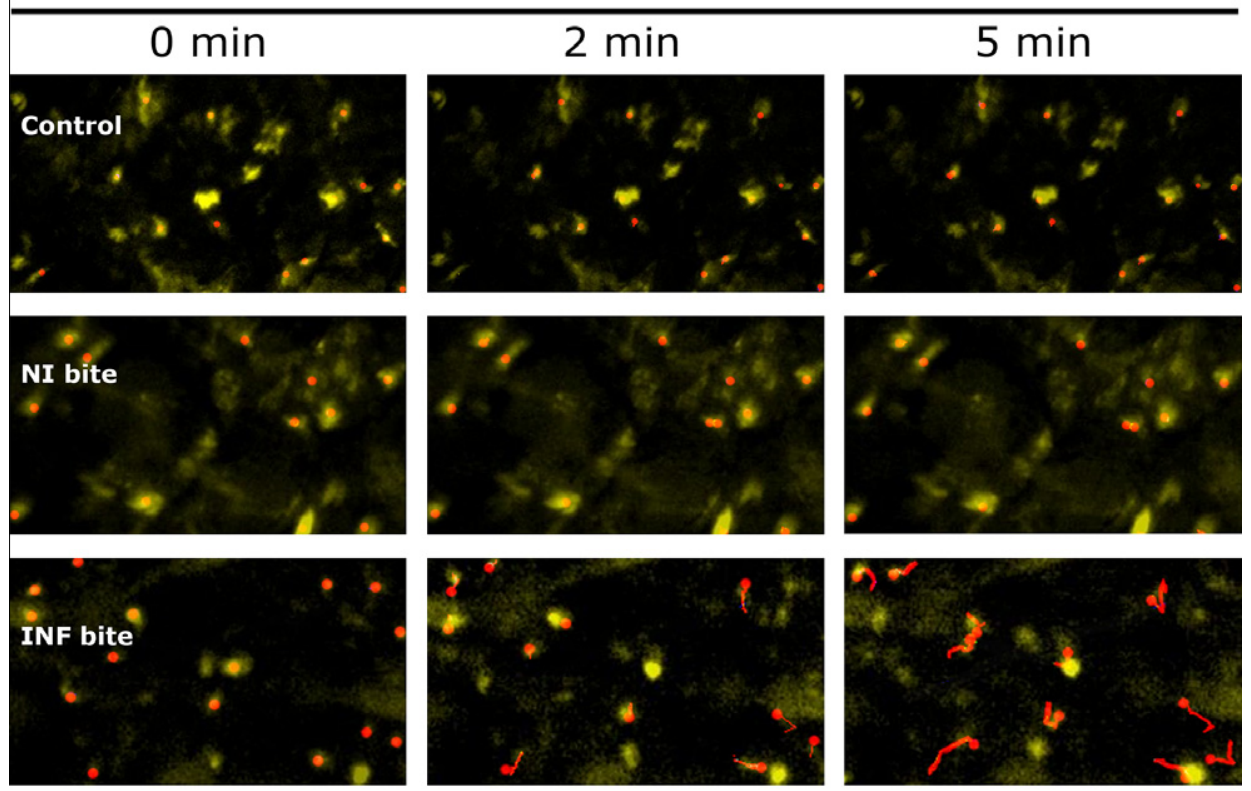

B

*

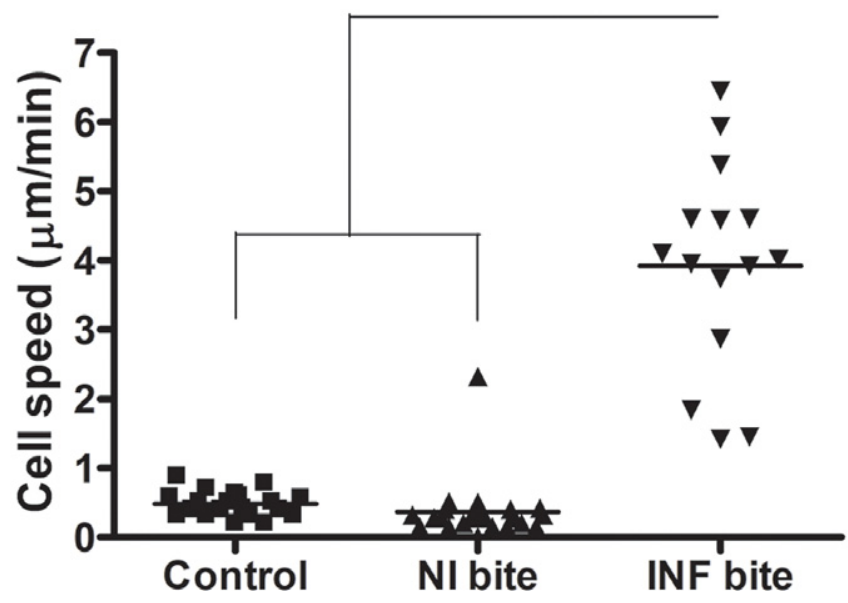

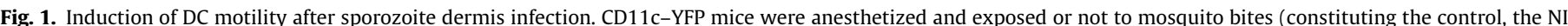

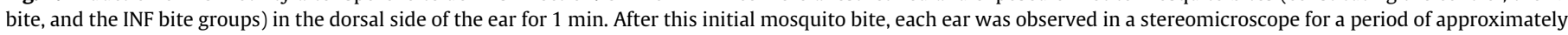

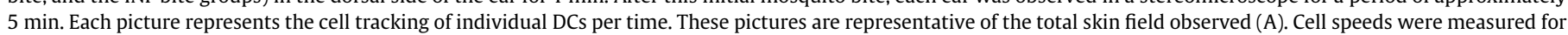
the cells from each group described above (B). 


\section{Results}

3.1. Skin-inoculation of sporozoites by mosquito bites induces early DC and Treg motility

Since the immunologic events involving Tregs occurring at the time of sporozoite injection remain to be elucidated, we observed whether the initial parasite injection into the dermis could trigger a local response from Tregs and/or DCs. To accomplish this, P. berghei ANKA-infected mosquitoes were allowed to feed on the ears of Foxp3-GFP (Tregs) or CD11c-YFP (DCs) mice, respectively. Control groups consisted of samples taken from ears of mice bitten by noninfected mosquitoes or mice that were not bitten at all. The results clearly show that infected-mosquito bites (INF bite) first trigger DC movement (within 1-5 min after the mosquito bite; see Fig. 1 and Supplementary movie S1) and later, Treg movement (within 5-20 min after the mosquito bite; see Fig. 2 and Supplementary movie S2) at the site of inoculation. Note that Tregs did not move in the first 5 min after mosquito bites (Supplementary movie S3) and DCs stopped moving 5 min after mosquito bites (Supplementary Fig. 1 and movie S4). Next, we tracked the DCs and Tregs cells from INF bites, by using time-lapsed imaging, and observed an average cell speed of $4 \mu \mathrm{m} / \mathrm{min}$ for each (Figs. 1 and $2 \mathrm{~B}$, respectively). These results are in stark contrast with cell speeds observed DCs and Tregs from the control groups, consisting of non-infected mosquito bite (NI bite), where we found average cell speeds of less than $0.5 \mu \mathrm{m} / \mathrm{min}$ (Figs. 1 and 2B). When the Treg cell speeds were measured $2 \mathrm{~h}$ after mosquito bites, there was no observed difference in cell speeds between the infected (INF) group and the non-infected (NI) group (data not shown). In order to remove human bias, an automatic algorithm was used to identify and track Tregs which confirms the results from manual identification and tracking (Supplementary Fig. 2, movie S5, and supplemental experimental procedures).

A

Time after mosquito bite
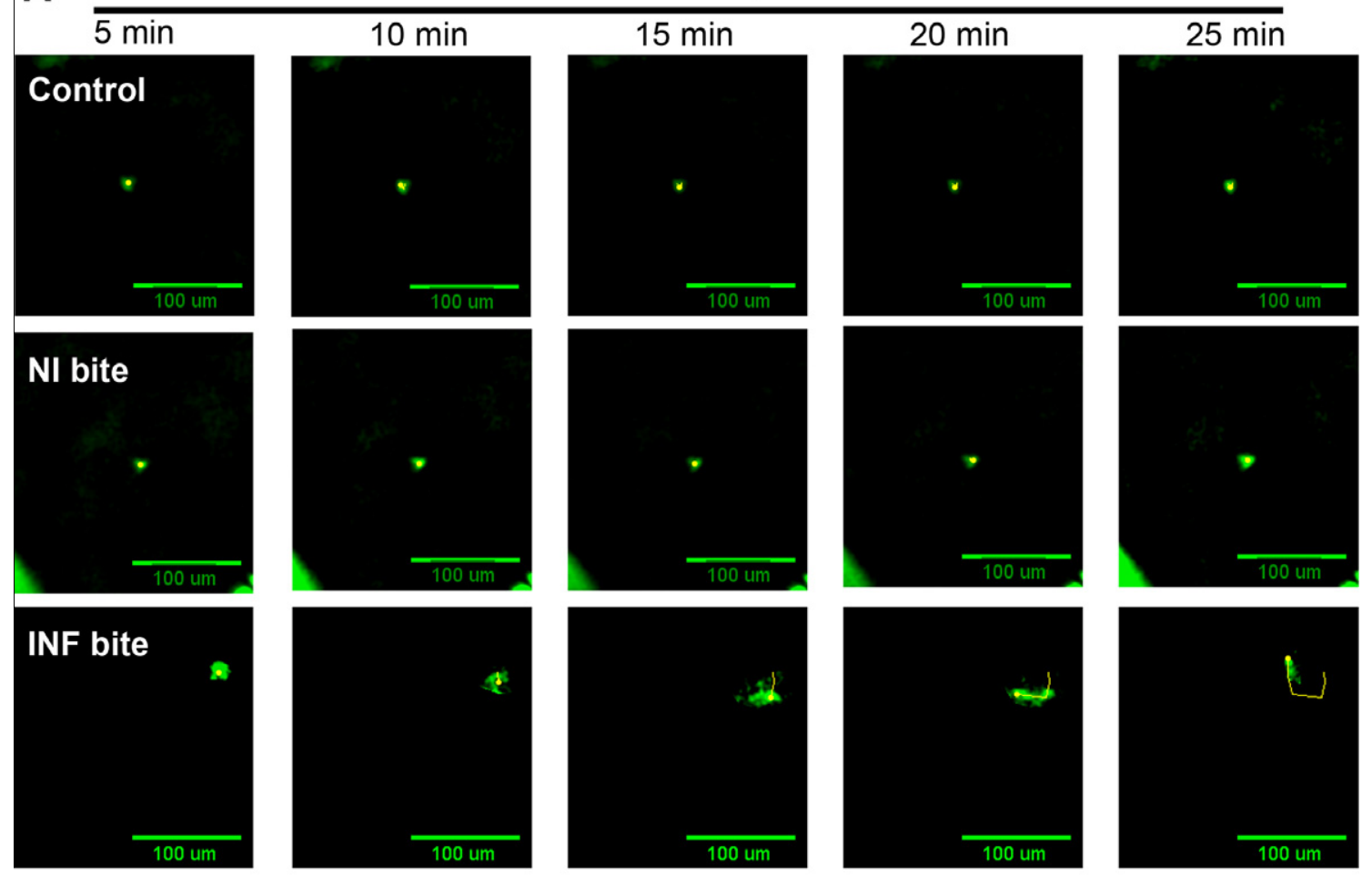

B

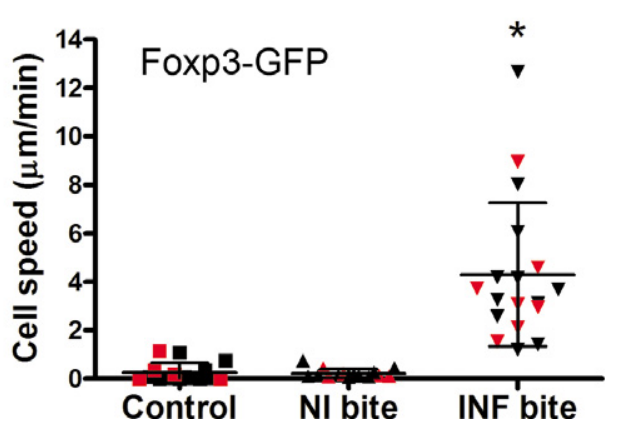

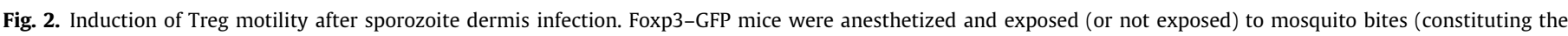

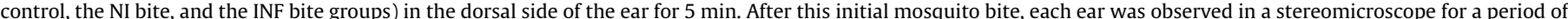

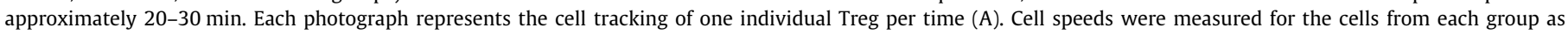
described above. The different colors in the figure represent cell speed from independent experiments (B). 
3.2. Alteration of CD62L and CTLA-4 expression in Tregs 30 min after mosquito bites

Since it appeared that infected mosquito bites only affect the behavior of DC and Tregs on the skin, we evaluated whether this was related to qualitative differences amongst DC and Tregs in those mouse groups. An increase in leukocyte infiltrates is evident in the skin after mosquito bites (either infected or non-infected), as compared to non-bitten control skins (Control group), measured through an observed percentage increase of $\mathrm{CD} 45^{+}$cells (data not shown). Despite the fact that the Treg percentage (CD4 ${ }^{+} \mathrm{CD} 45^{+}$ Foxp3-GFP ${ }^{+}$cells) was higher in the NI bite group (Fig. 3A, left), comparable numbers of Tregs were found between the NI bite and the INF bite groups (Fig. 3A, right). Next, we evaluated the phenotypic characteristics of these Tregs (Supplementary Fig. 3) and found that they had the same expression of CD25 and CD28 molecules (Fig. 3B). However, evaluation of the expression of CD62L showed that Tregs from NI bite groups showed a decrease in the expression of this molecule in comparison to Tregs from the control or the INF bite groups (Fig. 3B). The CTLA-4 expression in both the NI and the INF bite groups is lower when compared with the expression in control group (Fig. 3B). Finally, non-Tregs (CD4 ${ }^{+}$ CD45 ${ }^{+}$Foxp3-GFP ${ }^{-}$cells) showed no differences in the expression of these molecules described above (Fig. 3B). These results indicate that only skin Tregs from infected-mosquito bitten mice can keep their lymph node migration capacity (by maintenance of CD62L expression), and Tregs decrease their CTLA-4 expression when they are actively suppressing the immune response. Alternatively, since CD62L expression in Tregs can be correlated with loss of suppressive activity [17], Tregs from the INF bite group could be better suppressors than Tregs from NI or control groups.

\subsection{Decrease in MHC II and B7 expression on APCs 30 min after mosquito bites}

The experimental differences observed in Tregs amongst the different mouse groups used in our study may lead to quantita- tive and qualitative changes in the skin APCs. Therefore, we evaluated the skin APC infiltrates by flow cytometry after mosquito bites. Our results showed that B cell percentages and $\mathrm{CD} 11 \mathrm{C}^{+} \mathrm{B} 220^{+}$were very small and showed no difference amongst the groups (data not shown). The other APCs were divided in four groups, classified according to previous reports [18-20]: conventional/migrating DCs (cDCs/miDCs; CD11 $\mathrm{c}^{\text {high }}$ ), dermal/migrating DCs (DDCs/miDCs; CD11 $\mathrm{c}^{\text {low }}$ ), differentiating/migrating DCs (difDCs/miDCs; CD11 ${ }^{\text {low }} \mathrm{CD} 11 \mathrm{~b}^{+}$), and monocytes/macrophages (monocytes/M0s; CD11b ${ }^{+}$) (Supplementary Fig. 4A), and an increase in the percentage of $\mathrm{CDCs} / \mathrm{miDC}$ in the skin of INF bite group was observed as compared to the control or NI bite groups (Fig. 4A, left). Additionally, when we evaluated the amount of these CD11c subtypes (Fig. 4A, right), the numbers of cDCs/miDCs, DDCs/miDCs, DifDCs/miDCs were higher in the INF bite group than in the NI bite or the control groups (Fig. 4A, left). The monocytes/M0s numbers were increased in both mosquito bitten groups (NI bite or INF bite) as compared with the control group (Fig. 4A, left).

Given this evaluation of the APC percentages, we evaluated the expression of MHC class II, CD80, and CD86 molecules on these APCs subtypes. The expression of CD80 molecules showed no difference in these groups (Supplementary Fig. 4B). We could observe an increase in MHC class II expression on CDCs/miDCs of mosquito bitten animals (the NI bite and the INF bite groups) as compared with the control groups (Fig. 4B). However, in DDCs/miDCs the increase in MHC class II expression was only seen of the NI bite group (Fig. 4B). Monocytes/M0s from mosquito bitten groups (NI or INF bite) had a lower expression of MHC II expression in comparison with the control groups (Fig. 4B). The expression of the co-stimulatory molecule CD86 was decreased in cDCs/miDCs or DifDCs/miDCs from mosquito bitten groups (NI or INF bite) when compared with the control group (Fig. 4B). Thus, despite the fact that increased numbers of APCs were attracted to the parasite injection site, our results indicate that these cells had a low APC capacity by decreased expression of MHC class II or co-stimulatory molecules on their cell surface.

A

Gated on CD4+CD45+ cells:
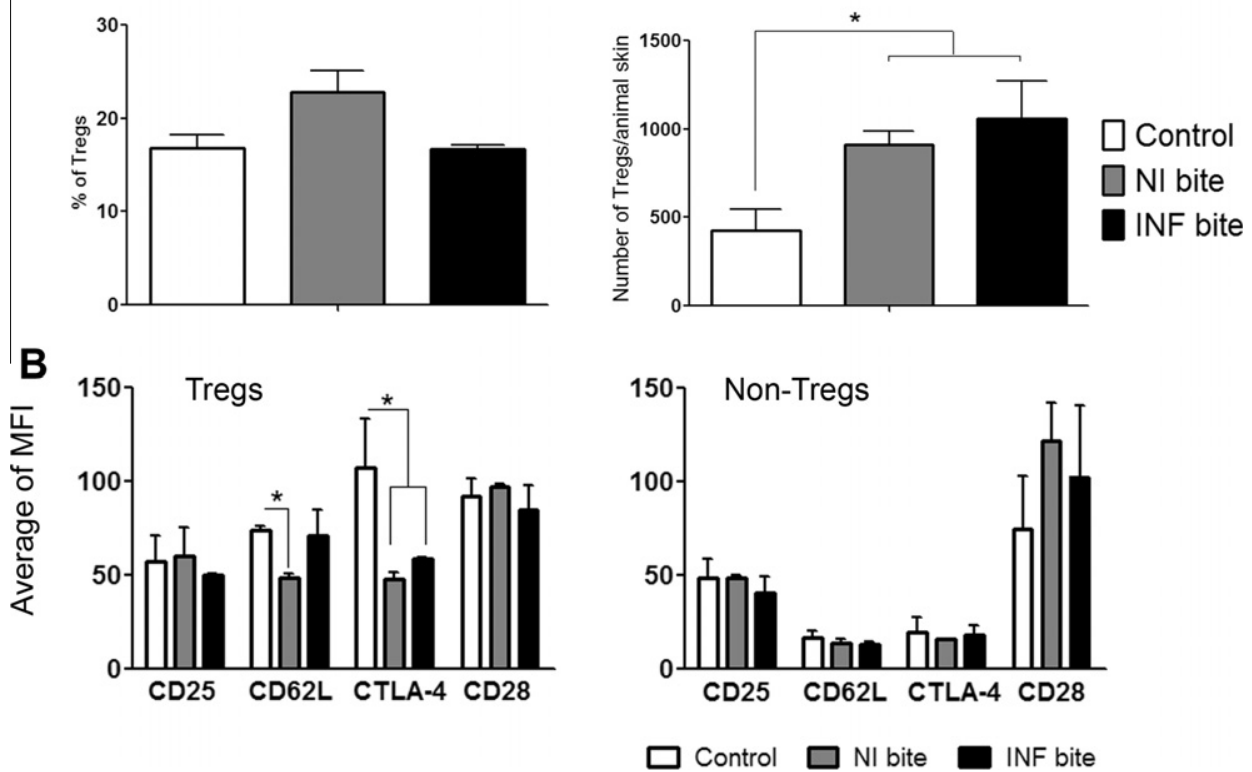

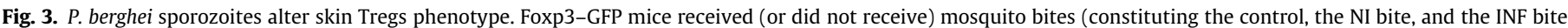

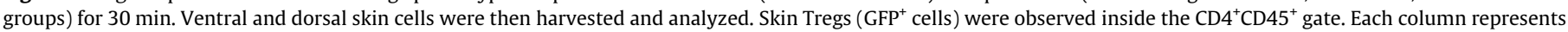

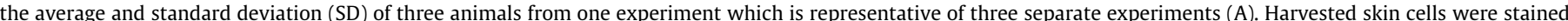

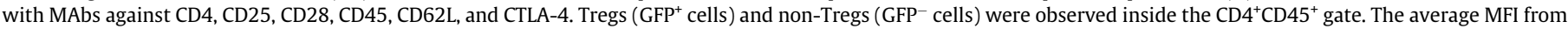
three animals analyzed per group from one experiment which is representative of three separate experiments (B). 

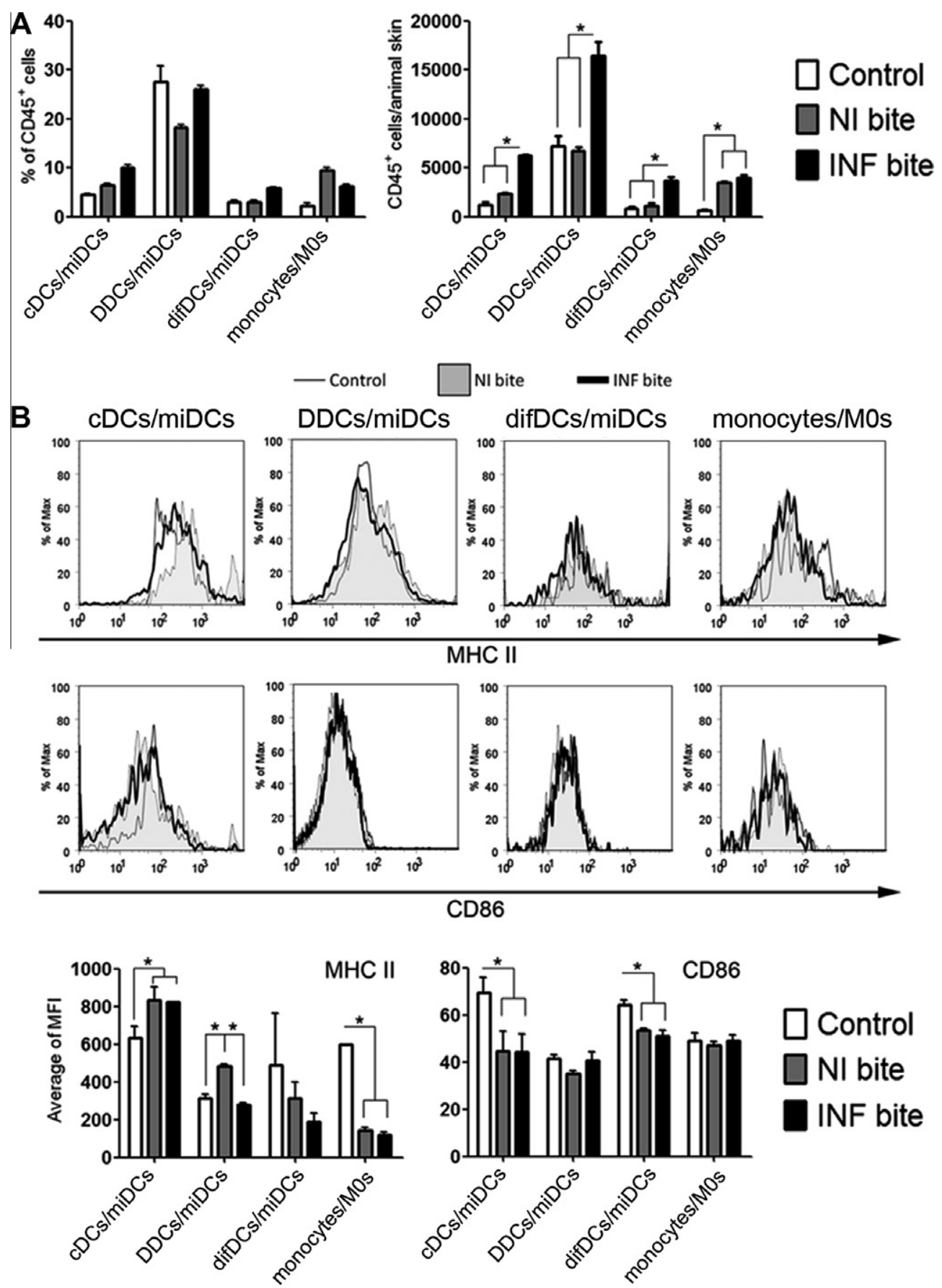

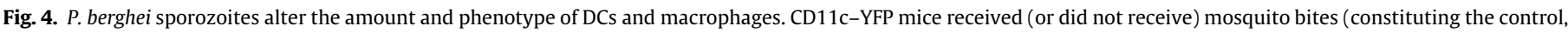

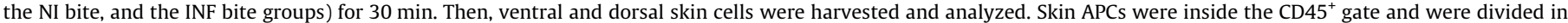

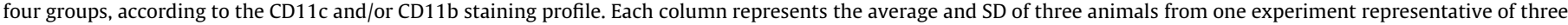

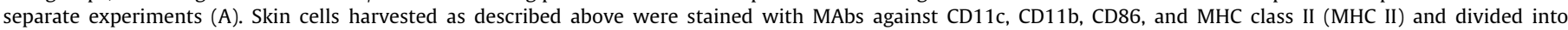

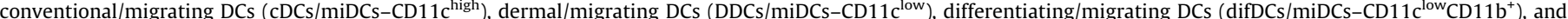

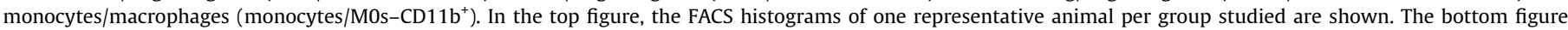
shows the average MFI from three animals analyzed per group from one experiment which is representative of three separate experiments (B).

\subsection{Phenotypic analysis of skin Tregs and non-Tregs $2 \mathrm{~h}$ after mosquito} bites

Our results establish early Treg and DC activity in the skin just after a mosquito bite. In particular, we observed that infected mosquito bites trigger DC and Treg motility, maintenance of CD62L expression on Tregs, and modulation on MHC class II or B7 molecules on DCs. We postulate that these changes could be related to a suppression of the immune system triggered by the parasite. To address this possibility, we studied the phenotype of T-cells found in skin $2 \mathrm{~h}$ after the different types of mosquito bites described previously (Fig. 5).

From this comparison, we found that the total number of CD4 T cells inside the skin was comparable between the NI and the INF bite groups, yet higher than that found in the control groups (Fig. 5A). There was, however, an observable difference found in 
the expression of surface molecules on Tregs and non-Treg cells (Fig. 5B). In particular, we found that Tregs from NI bite groups had the same amount of CD62L expression than Tregs from INF bite groups (Fig. 5B, left panel); however, they were both higher than the control groups. This indicates that both could have the same migration capability towards the draining LNs and/or the same activation level. However, the level of CTLA-4 expression in Tregs from the NI bite groups was higher than the INF bite groups (Fig. 5B, left panel). If CTLA-4 internalization occurs after Treg activation, this result could indicate a higher Treg activity in the INF bite groups than in the NI bite groups. The expression of CD62L, CTLA-4, and CD28 in non-Treg cells (Fig. 5B, right panel) shows that while non-Treg cells of the NI bite group exhibit decreased CD62L expression, the same cells from the control or the INF bite groups maintain expression of CD62L (Fig. 5B, right panel). This may indicate that non-Tregs of non-infected bitten skins do not migrate as well as their counterparts in infected bitten skins; moreover, it is an indicative that the non-Tregs from NI bite group had enhanced activation in comparison to INF bite group non-Tregs. The
non-Tregs from NI bite group also showed increased CD28 expression while the same cell type from the INF bite group does not. These results are strongly correlated to results of the CTLA-4 expression for these cells, since non-Tregs from the NI group exhibit decreased expression, while demonstrating increased expression in the INF groups (Fig. 5B, right panel). These CD28/CTLA-4 results appear to support the hypothesis that sporozoite skininjection may trigger an enhanced suppression of non-Tregs in the skin.

\subsection{Phenotypic analysis of draining lymph node (LN) Tregs and non-} Tregs $2 \mathrm{~h}$ after mosquito bites

Skin-draining LNs are one of the first "filters" to antigens originating from the skin. Thus, if the sporozoite triggers any type of suppression in the immune system, alterations of the LN cellular activation of $\mathrm{T}$ cells could provide indirect evidence supporting our hypothesis. As such, we observed the number of T cells in these LNs and found that the numbers of Treg and non-Treg were higher

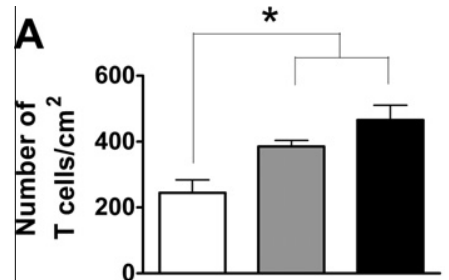

B

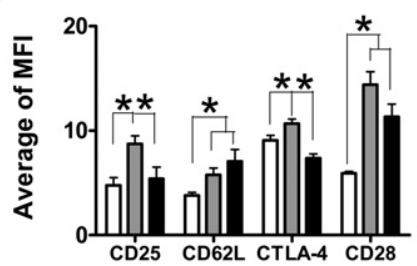

C

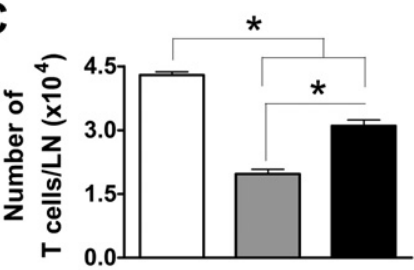

D

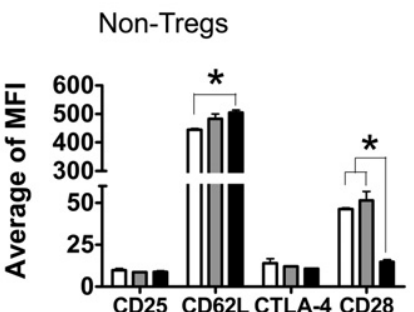

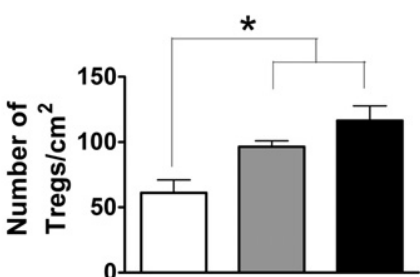

$\square$ Control $\square$ NI bite INF bite
Tregs
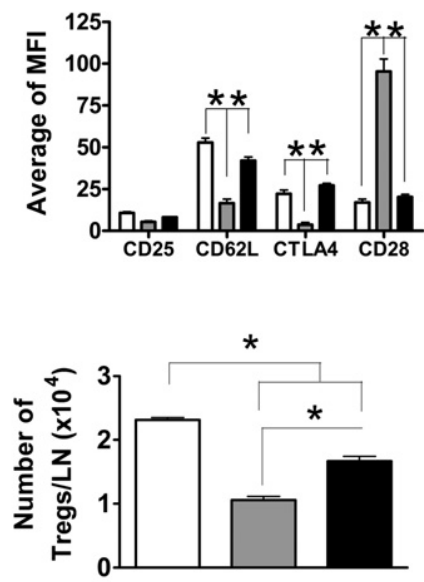

Tregs

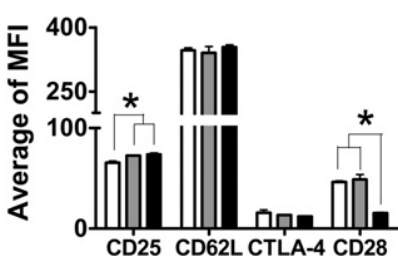

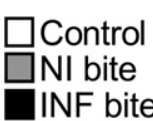


in draining LNs from the INF bite groups as compared with the NI bite groups (Fig. 5C). However, CD28 expression was also lower in $\mathrm{T}$ cells (Tregs and non-Tregs) from the INF bite groups than from the NI bite groups (Fig. 5D). Since CD28 is an activation marker, this result may be an indication that $\mathrm{T}$ cells were less activated in draining LNs from the INF bite groups.

\subsection{Phenotypic analysis of skin and draining LN DCs $2 \mathrm{~h}$ after mosquito bites}

From the studies described, T cells from the INF bite groups show a remarkable difference in their phenotype markers when compared with the control or the NI bite groups. These differences may be associated with differences in the APCs present within the skin. Therefore, we also evaluated the expression of MHC class II and B7 molecules (CD80 and CD86) on APCs isolated from these skins, $2 \mathrm{~h}$ after mosquito bites. Skins from the INF bite groups had more APCs than the control or the NI bite groups (Fig. 6A). Despite the fact that CD80 expression was higher in $\mathrm{CDCs} / \mathrm{miDCs}$ of the INF bite groups than the control or the NI bite groups (Fig. 6B), the expression of MHC class II molecules (Fig. 6B) or CD86 molecules (Fig. 6B) was lower or unchanged among all APCs. This could be indicative of a tolerogenic state of APCs from the INF bite groups. In fact, these APCs did not leave the skin since they were observed in small quantity in the draining LNs of the INF bite groups as compared with the quantity of these cells found in the control or the NI bite groups (Fig. 6C). The gates used to identify these APC populations were previously established (Supplementary Figure S3).

\subsection{Reduced homeostatic proliferation of skin-migrated CD4 T cells}

Since the numbers of Tregs within the NI or the INF bite skins are too small (approximately 100 cells $/ \mathrm{cm}^{2}$ ), it is not possible to perform an in vitro suppression assay in order to directly test these Tregs. Nonetheless, we tested their suppressive capacity by an indirect method, where we performed skin transplantations from these groups (Control, NI bite, and INF bite) to nude animals. In these immune-compromised nude animals, CD4 T cells expand by homeostatic proliferation, which can be suppressed by Tregs [16]. In our experiments, we observed that homeostatic proliferation was lower in recipients of INF bitten skins as compared with those of NI bitten skins (Fig. 7A), since the total number of CD4 T cells were reduced in spleens of recipients of skins from the INF bite group. This difference was not seen in inguinal (iLN) or mesentheric (mLN) lymph nodes (Fig. 7A), mostly because these differences only occur in LNs during the first days after transplantation. We still found higher expression of CD69 and CD62L in CD4 T cells from the INF bite groups as compared with that found in the control or the NI bite groups (Fig. 7B). Although the CD4 T cells taken from skins of the INF bite groups had a smaller homeostatic proliferation than CD4 T cells from the other groups, the number increased from their initial number of 200-300 T cells present within the skin transplant. Therefore, it was possible to associate the presence of activation markers such as CD69 and CD62L to this expansion.

\section{Discussion}

We observed interesting features concerning the behavior of skin Tregs and DCs, $30 \mathrm{~min}$ after the inoculation of sporozoites by infected mosquito bites. Our results show that local DCs and Tregs start to move after mosquitoes bites infected with $P$. berghei ANKA (Figs. 1 and 2; Supplementary Movies S1 and S2) and these Tregs exhibit a decreased expression of CTLA-4 as compared to the same expression levels measured in the control animals (Fig. 3).
A

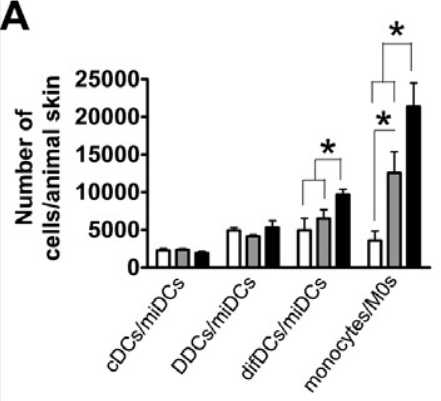

B

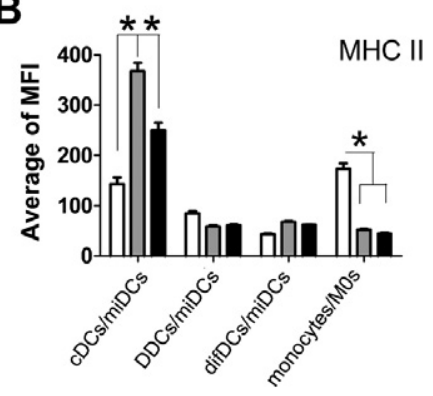

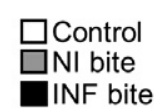
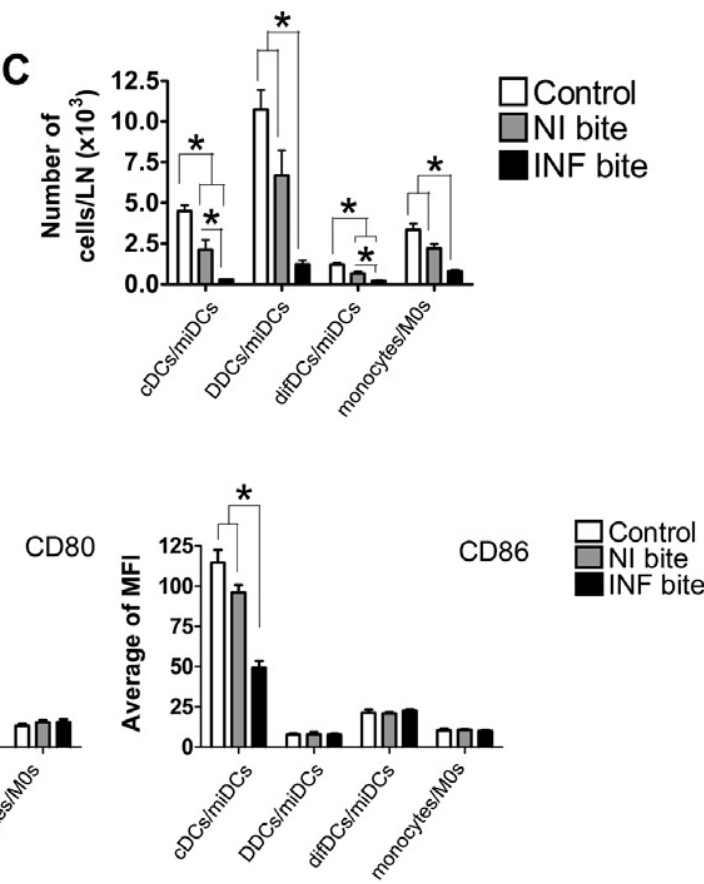

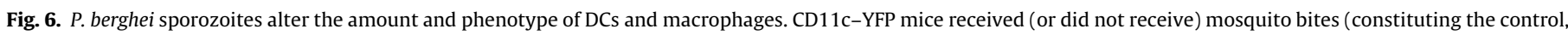

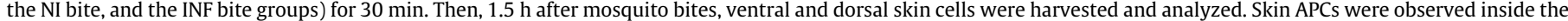

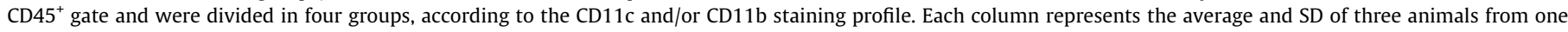

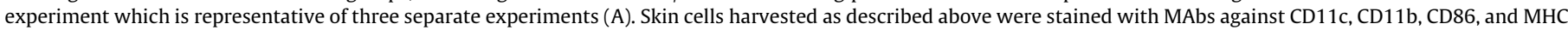

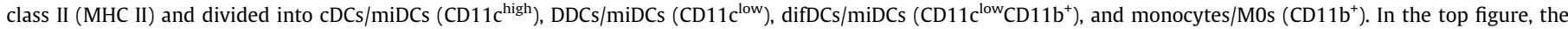

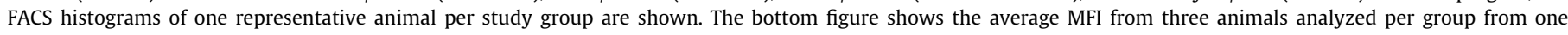

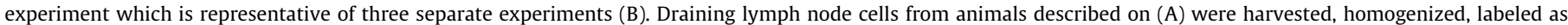

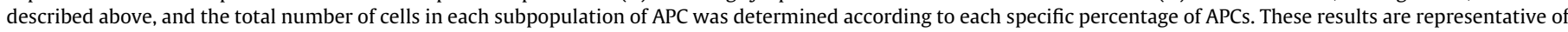
three separate experiments (C). 


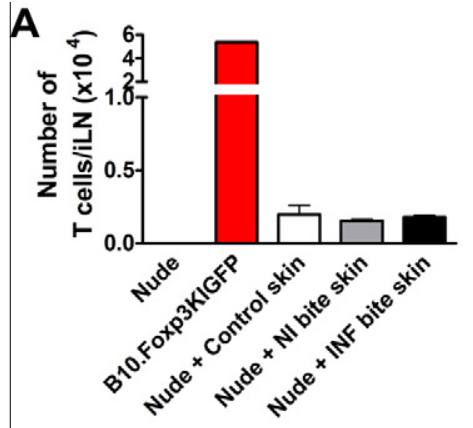

B

\section{Gated on CD3+CD4+ T cells:}
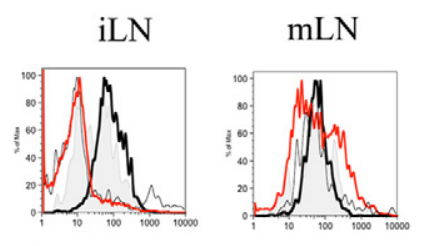

CD69
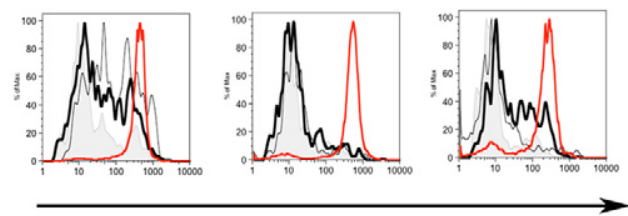

CD62L
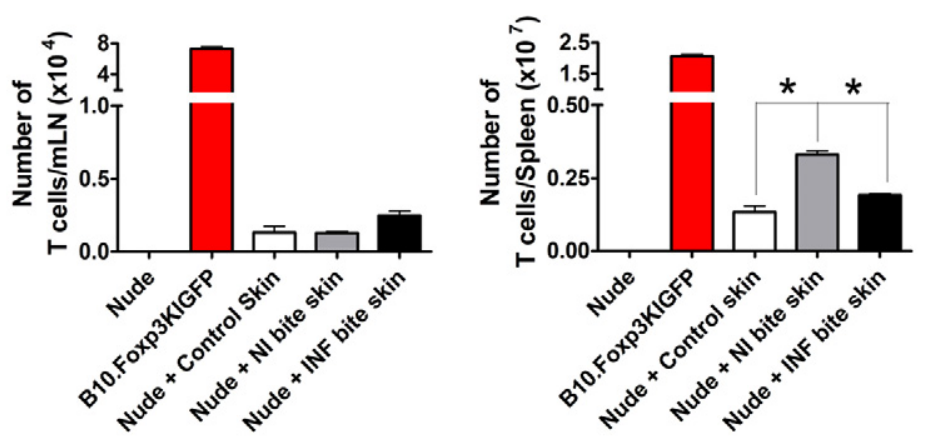

CD69
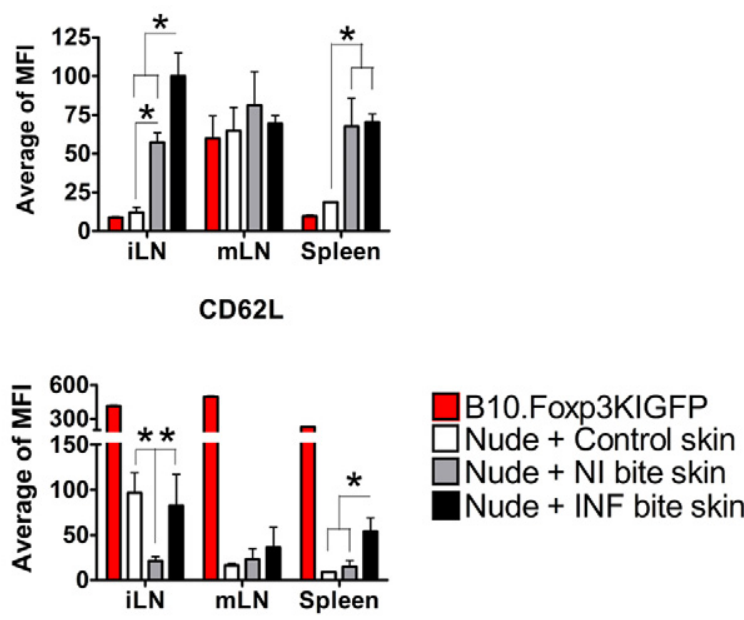

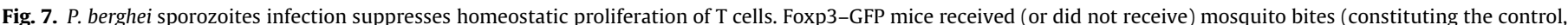

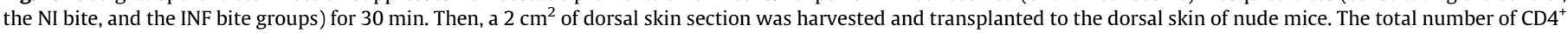

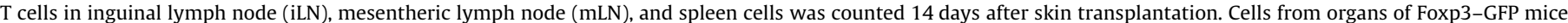

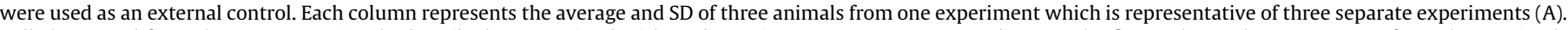

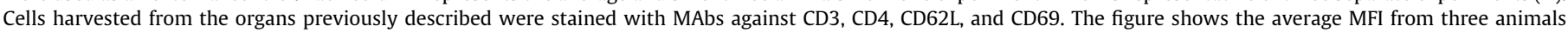
analyzed per group from one experiment which is representative of three separate experiments (B).

The same behavior was not observed in skin DCs or Tregs after non-infected mosquito bites. A decreased CTLA-4 expression level was previously described in activated cells [21,22], and it is known that CTLA-4 is essential for initial Treg in vivo suppressor activity on APCs $[23,24]$. According to our results, we suggest that rapid CTLA-4 internalization occurs in Tregs at the moment of contact with APCs, suppressing the expression of MHC II and/or B7 molecules on the later. Note, that we are not contradicting well-documented previous publications about crucial role of CTLA-4 in Treg function (e.g. [25]), but supposing that CTLA-4 could be internalized in a brief moment after Treg-APC contacts. Unfortunately, the amount of Tregs per $\mathrm{cm}^{2}$ of skin is approximately $50-100$ Tregs, which precludes the possibility of harvesting enough cells to perform in vitro suppression assay tests. However, we did address this suppressive capacity for in vivo experiments of homeostatic proliferation. Our results reveal that homeostatic proliferation of CD4 T cells, which migrated from skin to nude recipient mice, was attenuated for cases where skin transplants originated from the INF bite groups only (Fig. 7). Our skin phenotyping experiments of APCs, performed $30 \mathrm{~min}$ after mosquito bites, also reveal a decrease in the expression of MHC class II and CD86 molecules in APCs from skins of mice bitten by P. berghei ANKA-infected mosquitoes (Fig. 4), thereby corroborating the notion that Tregs are suppressing APC activation. Only Tregs from skins of infected-mosquito bites preserve their CD62L expression (Fig. 3), indicating that they could promptly migrate to the draining lymph node and further contribute to suppress the immune system activation. If this is the case, we should consider other aspects of parasite biology. Also, the maintenance of CD62L expression in Tregs is related to their active capacity to suppress immune responses [17] and, therefore, the maintenance of this L-selectin in INF bite Tregs (Fig. 3) could also indicate their active suppressive state.

The fact that infected mosquito bites (and not the non-infected mosquito bites) could trigger the mobilization of Tregs (Supplementary Movie S2) motivates further discussion of the role of Tregs in different situations when the skin homeostasis is broken. One may suppose that a "sterile" mosquito bite would only trigger responses to heal the epithelial disruption, for which Tregs would control only the activation of tissue scavengers, such as macrophages. In fact, our results demonstrate that non-infected mosquito bites increased only the amount of macrophages inside the tissue (Fig. 4A). However, when the parasite is present, the tissue destruction is persistent, with parasites destroying surrounding cells and inducing the release of different inflammatory mediators.

This phenomena just described appears to recruit different cell types to the bitten area, including DCs (Fig. 4A), which could be harmful to the parasite existence since DCs are better than macrophages at triggering adaptive immune responses [26]. However, we could also observe that these DCs had a decrease in their costimulatory capacity (decrease in CD86 expression, Fig. 4B) without, or even, increasing the expression of MHC class II (Fig. 4B). In this scenario, DCs could be better described as tolerogenic than immunogenic cells [27]. A determination as to whether Tregs start 
this process (since these differences could also be related to resident vs. recently migrated APCs) still needs to be established, but one could suppose that the continuous movement of skin Tregs after infected-mosquito bites would be an attempt to induce a tolerogenic phenotype on DCs and T cells in the skin, as well as in the draining $\mathrm{LN}$ after migration.

Observation of the DC and non-Treg results $2 \mathrm{~h}$ after the infected mosquito bite (Figs. 5-7) showed that DCs disappeared from dLNs (Fig. 7) and non-Tregs had decreased CD28 expression (Fig. 5D), thereby supporting the hypothesis that skin Tregs migrate towards the dLN in order to control immune system activation. Moreover, non-Tregs in the skin of the INF bite groups maintain high CTLA-4 expression and low CD28 expression, indicating a suppressed phenotype for these cells (Fig. 5B). Not only non-Tregs, but also the APCs in skins of the INF bite groups had a tolerized phenotype, since both MHC class II and CD86 expressions were compromised (Fig. 6B). All these results support evidence for an active suppressive state of the immune system by the sporozoite, potentially by activating Tregs, inducing a tolerogenic phenotype in DCs, or both.

In the course of parasite establishment within the host, malaria parasites must interact with the host environment, escape destruction by the host immune system, and at the same time avoid extended harm to the host until it is capable of transmission and continuation of its life cycle. Since Tregs have a central role in attenuating the immune system response against self antigens (e.g., autoimmunities) and exacerbated responses against non-self antigens (e.g., allergies) [4,5], they seem to be the perfect counter players for interaction by any successful parasite. By manipulating Treg functions, parasites can turn off exacerbated and misleading immune responses against self antigens which compromise both the host and parasite survival. An example of another protozoan parasite is Trypanosoma cruzi, which can completely suppress the development of Experimental Autoimmune Encephalomyelitis (EAE) and even suppress an ongoing EAE [28]. Thus, if as conjectured, sporozoites injection can only trigger an early activation of skin Tregs, they may contribute to the survival of the host until its life cycle is complete. Future studies should consider this hypothesis especially in the development of new vaccination approaches using this phase of the parasite life cycle [29]. Present vaccines that are already successful in boosting parasite immunity in mouse models and are in advanced clinical trials could be improved by the results obtained in our work.

In conclusion, this is the first time that infected mosquito bites have been shown to lead to specific immune response alterations involving Tregs and DCs inside the dermis and the draining lymph nodes. The experimental evidence accumulated in this paper provides a strong motivation for future research into DC and Treg response phenomena in skin with the infection of Plasmodium sporozoites. Our work supports the possibility that sporozoites have a direct impact in both DCs and Tregs. Furthermore, we demonstrate a mobilization of DCs and Tregs after infected mosquito bites which raises several questions concerning these skin cells' role in the establishment of infection and the involvement of the parasite and host molecules in this processes upon contact with the sporozoites. Thus, we believe that the data presented in this paper shall be important for future research into response mechanisms of skin DCs and Tregs in the presence of Plasmodium sporozoites.

\section{Acknowledgments}

We would like to thank Drs. Michael L. Dustin and Juan J. Lafaille for the critical review of this work. C57Bl/6.Foxp3-KI ${ }^{\text {gfp/gfp }}$ mice and C57Bl/6.CD11C-YFP animals were a generous gift from Drs. Vijay K. Kuchroo and Mohamed Oukka (Harvard University, USA) and Dr. Michel C. Nussenzweig (Rockefeller University, USA), respectively. Dr. Gabriel Martins (Faculdade de Ciências da Universidade de Lisboa, Portugal) and M.Sc. Francisco Henriques (Cell Imaging Unit, Instituto Gulbenkian de Ciência, Portugal) helped with the imaging analysis. Ms. Fernanda G. Baptista and Ms. Ana Parreira prepared all mosquitos used in this manuscript. This work was partially supported by the FCT grant PTDC/EBB-BIO/115514/ 2009 and H.B.S. was a recipient of a CAPES-FCT travel grant 258/ 2010.

\section{Appendix A. Supplementary data}

Supplementary data associated with this article can be found, in the online version, at http://dx.doi.org/10.1016/j.cellimm.2012.06. 003.

\section{References}

[1] World Health Organization, World Malaria, Report (2010).

[2] J. Langhorne, F.M. Ndungu, A.-M. Sponaas, K. Marsh, Immunity to malaria: more questions than answers, Nature immunology 9 (2008) 725-732.

[3] Y. Belkaid, Regulatory T cells and infection: a dangerous necessity, Nature reviews. Immunology 7 (2007) 875-888.

[4] S. Sakaguchi, F. Powrie, Emerging challenges in regulatory T cell function and biology, Science (New York, N. Y.) 317 (2007) 627-629.

[5] M.A. Curotto de Lafaille, N. Kutchukhidze, S. Shen, Y. Ding, H. Yee, et al., Adaptive Foxp3+ regulatory $\mathrm{T}$ cell-dependent and -independent control of allergic inflammation, Immunity 29 (2008) 114-126.

[6] A. Scholzen, G. Minigo, M. Plebanski, Heroes or villains? T regulatory cells in malaria infection, Trends in parasitology 26 (2010) 16-25.

[7] J.P. Vanderberg, U. Frevert, Intravital microscopy demonstrating antibodymediated immobilisation of Plasmodium berghei sporozoites injected into skin by mosquitoes, International journal for parasitology 34 (2004) 991-996.

[8] R. Amino, S. Thiberge, B. Martin, S. Celli, S. Shorte, et al., Quantitative imaging of Plasmodium transmission from mosquito to mammal, Nature medicine 12 (2006) 220-224

[9] M.M. Mota, G. Pradel, J.P. Vanderberg, J.C. Hafalla, U. Frevert, et al., Migration of Plasmodium sporozoites through cells before infection, Science (New York, N. Y.) 291 (2001) 141-144.

[10] R. Amino, D. Giovannini, S. Thiberge, P. Gueirard, B. Boisson, et al., Host cell traversal is important for progression of the malaria parasite through the dermis to the liver, Cell host \& microbe 3 (2008) 88-96.

[11] P. Gueirard, J. Tavares, S. Thiberge, F. Bernex, T. Ishino, et al., Development of the malaria parasite in the skin of the mammalian host, Proceedings of the National Academy of Sciences of the United States of America 107 (2010) 18640-18645.

[12] C. Kebaier, T. Voza, J. Vanderberg, Kinetics of mosquito-injected Plasmodium sporozoites in mice: Fewer sporozoites are injected into sporozoiteimmunized mice, PLoS pathogens 5 (2009) e1000399.

[13] S. Hori, T. Nomura, S. Sakaguchi, Control of regulatory T cell development by the transcription factor Foxp3, Science 299 (2003) 1057-1061

[14] R.A. Clark, T.S. Kupper, IL-15 and dermal fibroblasts induce proliferation of natural regulatory T cells isolated from human skin, Blood 109 (2007) 194202.

[15] K. Lahl, C. Loddenkemper, C. Drouin, J. Freyer, J. Arnason, et al., Selective depletion of Foxp3+ regulatory $\mathrm{T}$ cells induces a scurfy-like disease, The Journal of experimental medicine 204 (2007) 57-63.

[16] S. Shen, Y. Ding, C.E. Tadokoro, D. Olivares-Villagómez, M. Camps-Ramírez, et al., Control of homeostatic proliferation by regulatory $\mathrm{T}$ cells, The Journal of clinical investigation 115 (2005) 3517-3526.

[17] K.S. Goudy, M.C. Johnson, A. Garland, C. Li, R.J. Samulski, et al., Reduced IL-2 expression in NOD mice leads to a temporal increase in CD62Llo FoxP3+ CD4+ $\mathrm{T}$ cells with limited suppressor activity, European journal of immunology 41 (2011) 1480-1490.

[18] L. Eidsmo, R. Allan, I. Caminschi, N. van Rooijen, W.R. Heath, et al., Differential migration of epidermal and dermal dendritic cells during skin infection, Journal of immunology 182 (2009) 3165-3172.

[19] S. Henri, M. Guilliams, L.F. Poulin, S. Tamoutounour, L. Ardouin, et al., Disentangling the complexity of the skin dendritic cell network Immunology and cell biology 88 (2010) 366-375.

[20] M. Le Borgne, N. Etchart, A. Goubier, S.A. Lira, J.C. Sirard, et al., Dendritic cells rapidly recruited into epithelial tissues via CCR6/CCL20 are responsible for CD8+ T cell crosspriming in vivo, Immunity 24 (2006) 191-201.

[21] M.L. Alegre, P.J. Noel, B.J. Eisfelder, E. Chuang, M.R. Clark, et al., Regulation of surface and intracellular expression of CTLA4 on mouse T cells, Journal of immunology 157 (1996) 4762-4770.

[22] P.S. Linsley, J. Bradshaw, J. Greene, R. Peach, K.L. Bennett, et al., Intracellular trafficking of CTLA-4 and focal localization towards sites of TCR engagement, Immunity 4 (1996) 535-543. 
[23] Y. Onishi, Z. Fehervari, T. Yamaguchi, S. Sakaguchi, Foxp3+ natural regulatory cells preferentially form aggregates on dendritic cells in vitro and actively inhibit their maturation, Proceedings of the National Academy of Sciences of the United States of America 105 (2008) 10113-10118.

24] R.H. Friedline, D.S. Brown, H. Nguyen, H. Kornfeld, J. Lee, et al., CD4+ regulatory $\mathrm{T}$ cells require CTLA- 4 for the maintenance of systemic tolerance, The Journal of experimental medicine 206 (2009) 421-434.

[25] S. Read, V. Malmström, F. Powrie, Cytotoxic T lymphocyte-associated antigen 4 plays an essential role in the function of $\mathrm{CD} 25(+) \mathrm{CD} 4(+)$ regulatory cells that control intestinal inflammation, The Journal of experimental medicine 192 (2000) 295-302.
[26] R.M. Steinman, J. Banchereau, Taking dendritic cells into medicine, Nature 449 (2007) 419-426.

[27] S. Hugues, L. Fetler, L. Bonifaz, J. Helft, F. Amblard, et al., Distinct T cell dynamics in lymph nodes during the induction of tolerance and immunity, Nature Immunology 5 (2004) 1235-1242.

[28] C.E. Tadokoro, A.L. Vallochi, L.S. Rios, G.A. Martins, D. Schlesinger, et al., Experimental autoimmune encephalomyelitis can be prevented and cured by infection with Trypanosoma cruzi, Journal of autoimmunity 23 (2004) 103-115.

[29] S.T. Agnandji, B. Lell, S.S. Soulanoudjingar, J.F. Fernandes, B.P. Abossolo, et al., First results of phase 3 trial of RTS, S/AS01 malaria vaccine in African children, The New England journal of medicine 365 (2011) 1863-1875. 